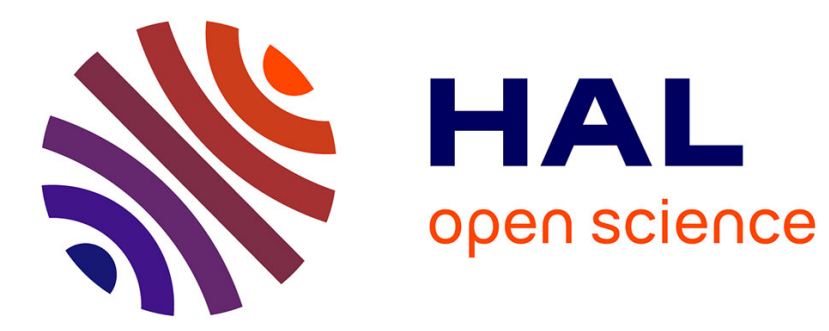

\title{
Interaction of heavy charged particles with atomic hydrogen
}

\author{
R. Lazauskas, J. Carbonell
}

\section{To cite this version:}

R. Lazauskas, J. Carbonell. Interaction of heavy charged particles with atomic hydrogen. European Conference on Few-Body Problems in Physics 18, Sep 2002, Bled, Slovenia. pp.167-170. in2p300012317

\section{HAL Id: in2p3-00012317 https://hal.in2p3.fr/in2p3-00012317}

Submitted on 23 Sep 2002

HAL is a multi-disciplinary open access archive for the deposit and dissemination of scientific research documents, whether they are published or not. The documents may come from teaching and research institutions in France or abroad, or from public or private research centers.
L'archive ouverte pluridisciplinaire HAL, est destinée au dépôt et à la diffusion de documents scientifiques de niveau recherche, publiés ou non, émanant des établissements d'enseignement et de recherche français ou étrangers, des laboratoires publics ou privés. 


\title{
Interaction of heavy charged particles with atomic hydrogen
}

\author{
R. Lazauskas* and J. Carbonell ${ }^{\dagger}$ \\ Institut des Sciences Nucléaires, 53 av. des Martyrs, 38026 Grenoble, France
}

\begin{abstract}
Faddeev equations in configuration space are employed to study low energy scattering of heavy positively charged particles on hydrogen atoms. Special interest is devoted to $\mathrm{pH}$ system, which was found to have a resonant value of scattering length, indicating the existence of a neartreshold $\mathrm{H}_{2}^{+}$bound state. Its binding energy $\mathrm{B}=1.13 \times 10^{-9}$ a.u. below $\mathrm{H}$ ground state, the smallest bound ever predicted.
\end{abstract}

The system of three charges constitutes a genuine 3-body problem studied from the birth of quantum mechanics. Since, much progress was done in solving bound state problem with explicit studies of molecular structures as $\mathrm{H}_{2}^{+}$, $p^{+} \mu^{-} p^{+}$, etc. On the other hand the rigorous solutions of three-body Coulomb scattering problem are still limited to the simplest cinematical cases as $e^{-}-\mathrm{H}$ and $e^{+}-\mathrm{H}$, where one particle is much heavier than others.

This contribution is devoted to extend these studies by considering elastic scattering of heavy positively charged particles, with mass $m$ in the range $m_{e} \leq m \leq m_{p}$, on atomic hydrogen at energies bellow inelastic thresholds. We will use all along the paper electronic atomic units $\left(m_{e}=e^{2}=\hbar=1\right)$.

The main difficulty of solving three-body Coulomb problem is related to long-range character of the potential. The primary equations of three body problem, the Faddeev equations, suppose free asymptotic behavior of the particles, in case of long range interaction become ill behaved due to noncompactness of their kernel. Anyway, they can still provide satisfactory solution for bound state problem when asymptotic conditions are implemented by vanishing the total wave function. They are however completely unapplicable for scattering states. The technical reason is that their right hand sides do not decrease fast enough to ensure the decoupling of Faddeev amplitudes in the asymptotic region and to allow unambiguous implementation of boundary conditions. In order to circumvent this problem, Merkuriev [1] proposed to split

*e-mail: lazauskas@isn.in2p3.fr

†e-mail: carbonel@isn.in2p3.fr 
the Coulomb potential $V$ into two parts (short and long range), $V=V^{s}+V^{l}$, by means of some arbitrary cut-off function $\chi$ :

$$
V^{s}(x, y)=V(x) \chi(x, y) \quad V^{l}(x, y)=V(x)[1-\chi(x, y)]
$$

One is then left with a system of equivalent equations

$$
\left(E-H_{0}-W_{\alpha}-V_{\alpha}^{s}\right) \Psi_{\alpha}=V_{\alpha}^{s} \sum_{\alpha \neq \beta} \Psi_{\beta} \quad W_{\alpha}=V_{\alpha}^{l}+V_{\beta}^{l}+V_{\gamma}^{l}
$$

with r.h.s. containing only short range contributions $\left(V_{s}\right)$ and with some 3body potential $\left(W_{\alpha}\right)$ which incorporates the long range parts. This approach was found very efficient in calculating the $\mathrm{e}^{+} \mathrm{Ps}$ and $\mathrm{e}^{+} \mathrm{H}$ cross sections $[3,4]$.

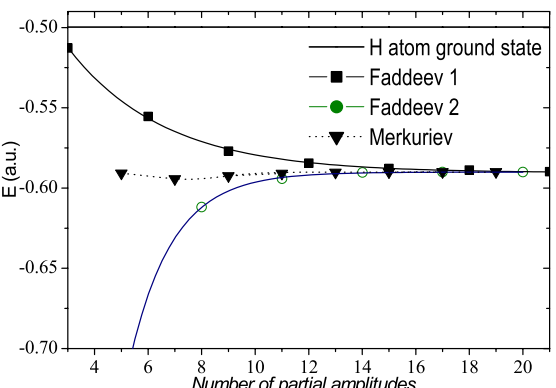

Figure 1. Convergence of Merkuriev and Faddeev equations in $\mu^{+} H$ ground state calculations.

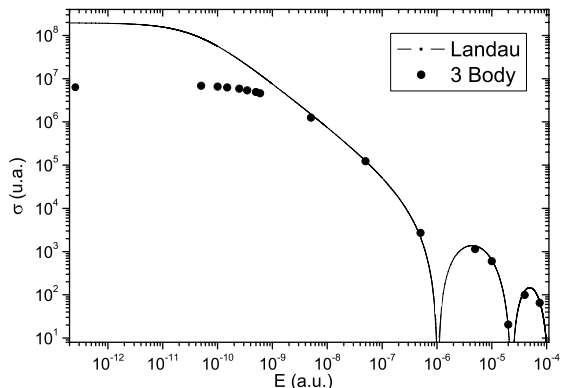

Figure 2. Low energy $\mathrm{pH}$ scattering in the pp triplet state, compared to the results of effective 2-body Landau potential.

Equations (2) were solved by expanding $\Psi_{i}$ in the bipolar harmonics basis and their components $\varphi_{i \alpha_{i}}$ in terms of two-dimensional splines. Its worth mentioning that the Merkuriev equations when applied to bound state problem are advantageous with respect to standard ones, because corresponding components are smoother functions and needs smaller bipolar harmonic basis to be described Fig.1. In this figure, two different convergence schemes of Faddeev equations are presented: Faddeev1 indicates a scheme where the number of the partial amplitudes is equal in all three components, whereas in Faddeev2 the number of the partial amplitudes in repulsive components is by 1 smaller than in attractive ones.

When dealing with a three-body Coulomb scattering problem some additional care should be taken when introducing boundary conditions - 2-body state is never free due to long range interaction - as well as when extracting the scattering observables from the asymptotic solution at finite distance. The long range polarization force makes the convergence of the phase shifts as a function of the $\mathrm{X}^{+}-\mathrm{H}$ distance very slow and requires an additional analysis [2]. 
Some interesting features of the 3-body Coulomb system can be learned from Fig.3, where the zero energy $\mathrm{X}^{+} \mathrm{H}$ cross section as a function of the projectile mass $m_{X}$ is displayed. Each peak corresponds to the appearance of a new S-wave bound state. The critical mass values $m_{i}$ at which they occur, enable to generalize the ground state stability triangle [5] to higher excitations. The calculated scattering lengths for physical projectiles $\mu^{+}$and $\pi^{+}$are $a_{\mu^{+} H}=69.1$ and $a_{\pi^{+} H}=24.4$ respectively. Other interesting feature about the $X^{+}-H$ system can be learned from studying the zero energy scattering wave functions. All the nodal structure of wave function is situated along the line connecting $\mathrm{X}^{+}$and $\mathrm{H}$ atoms center of mass. This corresponds to the "vibrational" bound states and contains information about their number.

Scattering of protons on $\mathrm{H}$ atoms presents special interest. The $\mathrm{pH} 3$-body wave function is antisymmetric with respect to the protons exchange. This can be realized in two different ways following the proton spin coupling. When two protons spins are antiparallel (singlet) the spatial part of the wave function is symmetric, while for the parallel case (triplet) it is antisymmetric. In BornOppenheimer approach, these two cases give rise to completely different 2-body effective potentials. The singlet case has a broad attractive well which supports a great number of bound states. They have been calculated since the first days of Quantum Mechanics and presently are known with a very high precision (see e.g. [6, 7] and reference therein). Our 3-body calculations cannot reach this kind of accuracy for bound states but are in 5-6 digits agreement for the lower excitations. Our calculations provide the first result for the $\mathrm{pH}$ scattering length $a_{s}=-29.3$. We notice that the zero energy scattering wave function shows 20 nodes in $X^{+} \mathrm{H}$-direction, indicating the existence of $20 \mathrm{~L}=0 \sigma_{g}$ energy levels for $\mathrm{H}_{2}^{+}$.

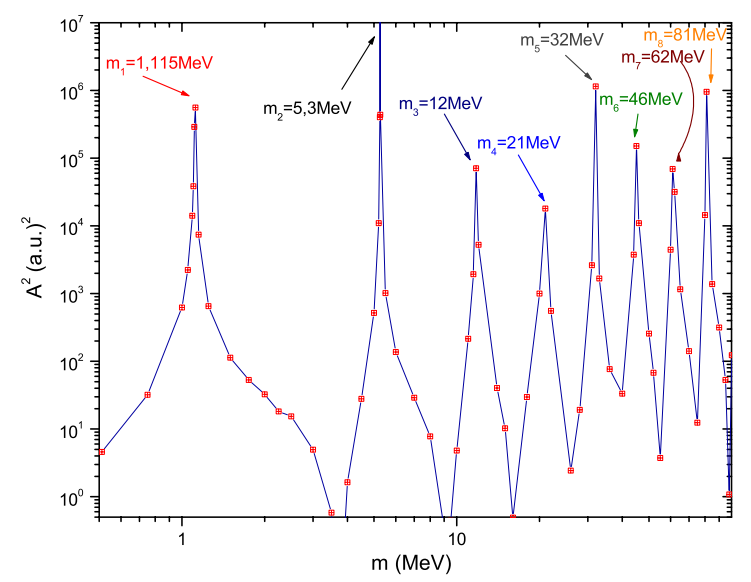

Figure 3. 3-body zero energy $\mathrm{X}^{+} \mathrm{H}$ cross section as a function of the projectile mass.

The triplet case - for which the 2-body potential was successfully modelled by Landau [9] - is dominated by the Pauli repulsion between the two 
protons, overbalanced at $r \sim 10$ by the attractive polarization forces. Our 3body calculations give a scattering length of $a_{t}=750 \pm 5$. The nodal structure of the Faddeev amplitudes indicates that such a big value is due to the existence of a first excited $\mathrm{L}=0$ state with extremely small binding energy. By using the modified effective range theory [10] we are able to determine its value $\mathrm{B}=(1.135 \pm 0.035) \times 10^{-9}$ below the $\mathrm{H}$ ground state. This result was recently confirmed by direct bound state calculation [11]. To our knowledge, this is the weakest bond ever predicted, three times smaller than ${ }^{4} \mathrm{He}$ atomic dimer [8].

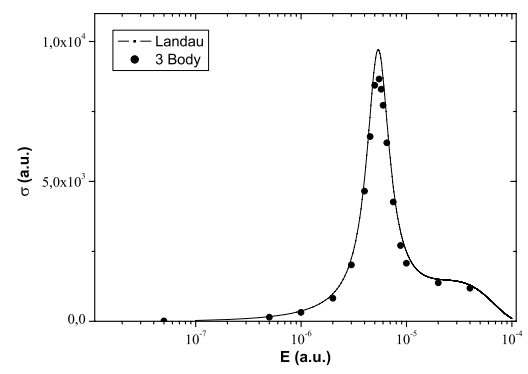

Figure 4. $\mathrm{pH}$ elastic cross section for $\mathrm{L}=3$ in the pp spin triplet state.

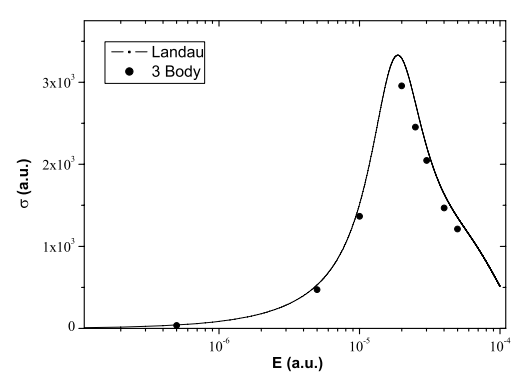

Figure 5. pH elastic cross section for $\mathrm{L}=4$ in the pp spin triplet state.

The $\mathrm{pH}$ cross sections for higher partial waves have also been calculated. They exhibit narrow resonances in several partial waves. Figs. 4-5 show the elastic cross sections for the $\mathrm{L}=3$ and $\mathrm{L}=4$ states. The position and width of these resonances were estimated to be $\mathrm{E}=(5.13-1.61 i) \times 10^{-6}$ for $\mathrm{L}=3$ and $\mathrm{E}=(1.56-0.94 i) \times 10^{-5}$ for $\mathrm{L}=4$.

Acknowledgement. We are grateful to C. Gignoux for useful discussions. Numerical calculations were performed at CGCV (CEA Grenoble) and IDRIS (CNRS). We thank the staff members of these organizations for their support.

\section{References}

1. Merkuriev, S.P. : Ann. Phys. 130, 395 (1980)

2. R. Lazauskas, J. Carbonell: Few-Body Systems 31, 125 (2002) and references therein

3. A. A. Kvitsinsky, J. Carbonell, C. Gignoux: Phys Rev. A46 (1992) 1310

4. A. A. Kvitsinsky, J. Carbonell, C. Gignoux: Phys. Rev. A51 (1995) 2997

5. A. Martin, J.-M. Richard and T.T. Wu, Phys. Rev. A46 (1992) 3697

6. Taylor, J.M., Yan, Z., Dalgarno, A., Babb, J.F.: Molec. Phys. 97, 25 (1999)

7. Hilico, L., Billy, N., Gremaud, B., Delande, D.: Eur. Phys. J. D12, 449 (2000)

8. F. Luo, G. McBane, G. Kim, C. Giese, W. Gentry, J. Chem. Phys. 983564 (1993)

9. Landau, L., Lifshits, E.: Mecanique Quantique, Ed. Mir Moscou 1975,

10. O’Malley T.F., Spruch L., Rosenberg L., J.: Phys. Rev. 125491 (1961)

11. J. Carbonell, R. Lazauskas, D.Delande, L. Hilico, S. Kilic: physics/0207007 (2002) 